Research Article

\title{
Protection against laryngeal and pharyngeal carcinoma: Heterozygous vs. homozygous deletions of GSTM1 and GSTT1
}

\author{
Nosheen Masood and Mahmood Akhtar Kayani \\ Cancer Genetics Laboratory, Department of Biosciences, COMSATS Institute of Information Technology, \\ Islamabad, Pakistan.
}

\begin{abstract}
Deletions in GSTM1 and GSTT1 genes are considered to be a risk factor for cancer development but the exact location of these deletions in the genome was unknown. Three main objectives of the current study were to: (a) identify the boundaries of these deletions in the human genome, (b) screen homozygous (-/-) and heterozygous (+/-) deleted, as well as homozygous present (+/+) individuals using PCR assays, (c) detect associations of pharyngeal (PC) and laryngeal cancer (LC) with the respective genotypes. In total, 102 PC and 92 LC patients were screened and compared with 150 controls. PCR mapping and sequencing revealed a $6 \mathrm{kbp}$ deletion for GSTM1 and a $9 \mathrm{kbp}$ deletion for the GSTT1 gene. The mean age of PC cases was 48.1 ( \pm 16.7$)$ years; for LC cases it was $48.5( \pm 17.4)$ years and for controls $46( \pm 17.7)$ years. The OR (odds ratio) for the GSTM1 null genotype in PC and LC cases was 10.2 and $1.0(95 \% \mathrm{Cl} 5.04-20.7$ and $1.1-1.7)$ respectively. Similarly, for GSTT1 the OR was 4.02 with a $95 \% \mathrm{Cl}$ of $2.3-7.1$ in PC cases. For LC cases the OR was 0.8 with $95 \% \mathrm{Cl}$ of $0.4-1.7$. A non-significant number of $\mathrm{LC}$ and $\mathrm{PC}$ patients had heterozygous deletions of GSTM1 compared to controls (OD 0.5, 95\% Cl 0.2- 1.6 and OR 0.5, 95\% $\mathrm{Cl}$ 0.2- 1.5 respectively). The GSTT1 gene also showed a non-significant association in PC (OD 0.9, 95\% Cl 0.4-1.9), as well as in LC patients (OD $0.7,95 \% \mathrm{Cl} 0.3-1.7)$. The homozygous genotype was significantly associated with $\mathrm{PC}$ and LC, whereas the heterozygous was not so. The GSTM1 (-/-) and GSTT1 (-/-) genotypes are a risk factor for LC and PC, whereas the (+/-) genotypes are not.
\end{abstract}

Keywords: GSTM1, GSTT1 heterozygous, homozygous, deletions, cancer.

Received: March 13, 2012; Accepted: August 3, 2012.

\section{Introduction}

Larynx and pharynx cancers are fast growing tumors, characterized by high frequency of nodal and distant metastasis involvement. These cancers are common in southeast Asian populations (Lin et al., 2002). Numerous epidemiological studies have indicated that carcinogen metabolizing genes play an important role in determining individual susceptibility to cancer (Toru et al., 2008). Phase II Glutathione S transferase genes (GSTM1 and GSTT1), code for enzymes that are crucial in carcinogen detoxification processes. They mediate conjugation of electrophilic compounds with reduced glutathione and facilitate excretion of the respective carcinogens. Glutathione S-transferase mu-1 (GSTM1) and glutathione S-transferase theta-1 (GSTT1) polymorphisms partially explain inter-individual variability in cancer susceptibility (Sinnett et al., 2000). Normal and malignant squamous cells of the larynx have been shown to express GSTM1 isoform in the highest concentra-

Send correspondence to Nosheen Masood. Cancer Genetics Laboratory, Department of Biosciences, COMSATS Institute of Information Technology, 46000 Park Road, Islamabad, Pakistan. E-mail: nosheenmasood@ hotmail.com. tion compared with GSTT1 (Bongers et al., 1995). Two alleles have been identified at the GSTT1 locus, one functional and the other nonfunctional (Pemble et al., 1994).

Until now, most studies concerning laryngeal and pharyngeal cancer relied on null (-/-) vs normal (+/+) genotype screening and significant associations between the GSTM1 null genotype and cancer predisposition are reported in the literature (Toru et al., 2008). However contradictory results were also found in case-control study analyses reporting non-significant associations between the presence of the GSTM1 null genotype and an increased risk for laryngeal (Gajecka et al., 2005) and pharyngeal (Cheng et al., 1999) cancer. For GSTT1, contradictory results have also found and not deteced any apparent role in the etiology of these cancers (Gajecka et al., 2005; Boccia et al., 2008). However, in different studies reviewed by Toru et al. (2008) significant associations have been reported between the GSTT1 null genotype and laryngeal (Boccia et al., 2008) and pharyngeal (Acar et al., 2006) cancer.

Whether GSTM1 or GSTT1 polymorphisms are a risk factor for laryngeal and pharyngeal cancer remains largely uncertain and a subject of research. In Pakistan, the relationship between GSTM1 and GSTT1 null genotype 
has been studied in normal population (Deng et al., 2004) and also on glaucoma patients (Shaikh et al., 2010). No data is available for these gene mutations and the risk of susceptibility to laryngeal and pharyngeal cancer in this population.

The current case-control study was designed with three main objectives: (1) GSTM1 and GSTT1 deletion mapping, (2) genotyping cancer patients $(+/+,+/-,-/-)$, and (3) to determine associations of the respectrive genotypes with pharyngeal and laryngeal cancer.

\section{Materials and Methods}

The present case-control study consisted of 102 pharyngeal and 92 laryngeal cancer cases that were pathologically confirmed by oncologists, as well as 150 cancer-free normal healthy individuals as controls. The cancer cases were recruited from the Nuclear Oncology and Radiotherapy Institute (NORI), Allied Hospital Faisalabad and the Pakistan Institute of Medical Sciences (PIMS) Islamabad, from November 2008 to March 2010, with prior approval from the Ethical Committees of both university and hospitals. All patients and normal individuals participated on a volunteer basis with consent. All subjects were personally interviewed according to a structured questionnaire. Blood was collected from both patients and controls in EDTA blood vacutainers and stored in $-20^{\circ} \mathrm{C}$ freezers until further use.

\section{DNA isolation and electrophoresis}

DNA was isolated, using a phenol-chloroform protocol (Khan et al., 2010). Isolated DNA was electrophoresed on $1 \%$ agarose gels and stained with ethidium bromide. Electrophoresis results were analyzed in a gel documentation system (BioDocAnalyze Biometra) and photographed. DNA quality was determined spectrophotometrically.

\section{Multiplex PCR}

Primers for GSTM1, GSTT1 exons and CYP1Al $\left(7^{\text {th }}\right.$ exon as internal control to check quality of DNA) were synthesized by using Primer 3 input software version 0.4.0 and BLAST using NCBI PRIMER BLAST (Table 1). Multiplex PCR assays were performed with $10 \mathrm{ng} / \mu \mathrm{L}$ DNA $(2 \mu \mathrm{L})$ added to a $20 \mu \mathrm{L}$ PCR mixture composed of $2 \mu \mathrm{L}$ PCR buffer, $10 \mathrm{mM}$ of each primer $(2 \mu \mathrm{L}), 25 \mathrm{mM}$ deoxynucleotide triphosphate $(0.24 \mu \mathrm{L})$ and $5 \mathrm{U} / \mu \mathrm{L}$ Taq polymerase $(0.2 \mu \mathrm{L})$. The reaction was run in a 9700 thermal cycler with the following protocol: $5 \mathrm{~min}$ at $94{ }^{\circ} \mathrm{C}, 30 \mathrm{cy}-$ cles for $25 \mathrm{~s}$ at $94{ }^{\circ} \mathrm{C}, 1 \mathrm{~min}$ at $72{ }^{\circ} \mathrm{C}$, followed by a final elongation step at $72{ }^{\circ} \mathrm{C}$ for $10 \mathrm{~min}$.

\section{Deletion detection}

Samples that failed to show amplification on $2 \%$ agarose gels were considered as homozygous deleted for the respective gene. Deletion-specific primers for GSTM1
Table 1 - Primer sequences used for GSTM1, GSTT1 and CYP1A1.

\begin{tabular}{|c|c|c|}
\hline Primer & Primer sequence & $\begin{array}{c}\text { Product } \\
\text { size }\end{array}$ \\
\hline GSTM1 $1 \mathrm{~F}$ & GCGGGAGGAAGTCTTACTGA & 371 \\
\hline GSTM1 $1 \mathrm{R}$ & ACACCCCCAACACACACAC & \\
\hline GSTM1 $2 \mathrm{~F}$ & GCTTCCCTGGTGCAGACA & 231 \\
\hline GSTM1 $2 \mathrm{R}$ & GCAGAGGCAGCCACAGGT & \\
\hline GSTM1 $3 \mathrm{~F}$ & TCCACCTGTCTCAGGGATCT & 240 \\
\hline GSTM1 $3 \mathrm{R}$ & TAAGCTGGGGAGAGGAGATG & \\
\hline GSTM1 $4 \mathrm{~F}$ & CATGTGACAGTATTCTTATTTCAGT & 298 \\
\hline GSTM1 $4 \mathrm{R}$ & ACTCAATCTCAGCATCACAGC & \\
\hline GSTM1 $5 \mathrm{~F}$ & GCAAGCACAACCTGTGTGAG & 250 \\
\hline GSTM1 $5 \mathrm{R}$ & TGTGCAGGAATGCAAGAGTC & \\
\hline GSTM1 $6 \mathrm{~F}$ & AGTTCCAGCTTGGGGAAGAT & 297 \\
\hline GSTM1 $6 \mathrm{R}$ & CCAAGAATATGTGGGCTGGA & \\
\hline GSTM1 7 F & ATGGTTTGCAGGAAACAAGG & 293 \\
\hline GSTM1 $7 \mathrm{R}$ & TCCAGGACTGGGAAAACATC & \\
\hline GSTM1 $8 \mathrm{~F}$ & GTGTCTGCAGTGGGGTTGT & 697 \\
\hline GSTM1 $8 \mathrm{R}$ & AGTCCCTTGGAAGAGGCAGT & \\
\hline GSTT1 $1 \mathrm{~F}$ & CCCGCAATTGGACTAAAGAG & 400 \\
\hline GSTT1 $1 \mathrm{R}$ & CTCCAAACCAGACCAGCAAT & \\
\hline GSTT1 $2 \mathrm{~F}$ & GCAGACTGGTGGGAAGAAGA & 300 \\
\hline GSTT1 $2 \mathrm{R}$ & TGCCTCTGAAGACTTTAGTTTCCT & \\
\hline GSTT1 $3 \mathrm{~F}$ & CAGAGCGAGACTCCGTATCA & 390 \\
\hline GSTT1 $3 \mathrm{R}$ & CAATTTGGCACAACAGAGGA & \\
\hline GSTT1 $4 \mathrm{~F}$ & GGCGAGAGAGCAAGACTCAG & 385 \\
\hline GSTT1 $4 \mathrm{R}$ & GGCAGCATAAGCAGGACTTC & \\
\hline GSTT1 $5 \mathrm{~F}$ & ATCTGTGGTCCCCAAATCAG & 632 \\
\hline GSTT1 $5 \mathrm{R}$ & GGGGGTTGTCTTTTGCATAG & \\
\hline CYP1A1 $7 \mathrm{~F}$ & TGTCTACCTGGTCTGGTTGG & 600 \\
\hline CYP1A1 $7 \mathrm{R}$ & CCTCCAGGACAGCAATAAGG & \\
\hline GSTM1 Del.up F & CGTTAGGATCTGGCTGGTGT & 200 \\
\hline GSTM1 Del.up R & GGGGCTGCACTCAGTAAGAC & \\
\hline GSTM1 Del.do F & CCTGGATGTCCCATTCATTC & 179 \\
\hline GSTM1 Del.do R & AGATTGGGTCCTGGAGACCT & \\
\hline GSTT1 Del.up F & GGCTGACACACTTTCAGTGG & 235 \\
\hline GSTT1 Del.up R & AGTGCCATCTATCGCATTCC & \\
\hline GSTT1 Del.do F & GGGGGTTGTCTTTTGCATAG & 396 \\
\hline GSTT1 Del.do R & CCCAGGCTGGAGTGCAGTGG & \\
\hline
\end{tabular}

and GSTT1 genes were designed from noncoding sequences flanking the genes. Primers were designed using Primer 3 software and BLAST online at NCBI. One set of primer corresponded to an upstream promoter region (primer del up F, primer del up R) and one set to a downstream non-coding region (primer del do $\mathrm{F}$, primer del do R) (Table 1). PCR assays were optimized separately with these two sets of primers. Then the forward primer of the upstream sequence (primer del up F) and the reverse primer 
of the downstream one (primer del do) were used to amplify genomic DNA (Figure 1). CYP1A1 was used as positive control.

\section{Electrophoresis}

Amplified products were resolved on $2 \%$ ethidium bromide-stained agarose gels along with a 100 bp DNA ladder. The photographs of gel electrophoresis were read by two technicians blind to each other's assessments.

\section{Sequencing}

The amplified products were sequenced by Macrogen (Korea). Both forward and reverse primers were used for sequencing in order to countercheck and confirm the results.

\section{Genotype status}

A multiplex PCR was performed with deletionspecific and exonic primers. Two bands in the electrophoretic gel corresponded to a heterozygous status whereas a single band revealed a homozygous genotype, as shown in Figure 2.

\section{Statistical analysis}

Statistical analyses for calculating OR, CI and standard deviations were done by using SPSS statistics 17.0 software and GraphPad Prism 5.

\section{Results}

The current study was based on the idea of mapping the deletion of GSTM1 and GSTT1 genes. These deletions were later on investigated for association with pharyngeal and laryngeal cancers. A total of 344 individuals, compris-

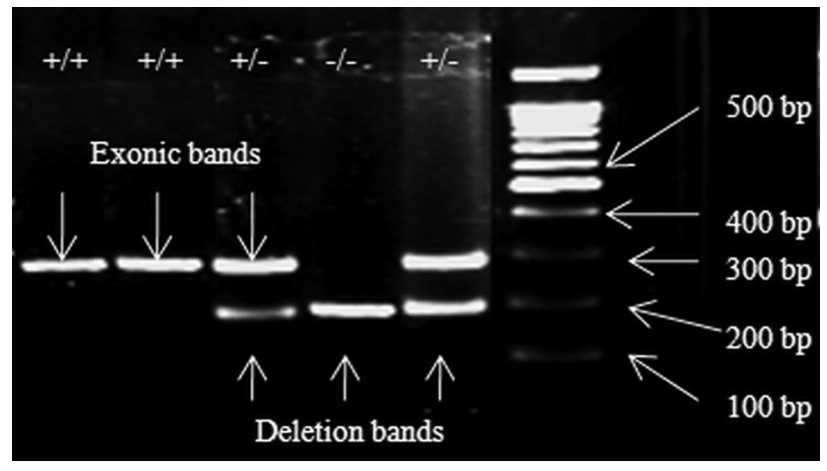

Figure 2 - Agarose gel electrophoresis results showing exonic and deletion-specific bands corresponding to homozygous present $(+/+)$, heterozygous (+/-) and homozygous deleted gene (-/-) samples.

ing 102 patients with confirmed pharyngeal cancer, 92 laryngeal cancer cases, and 150 normal healthy controls were included in the study. Mean age of pharyngeal cancer patients was $48.1( \pm 16.7)$ years, that of laryngeal cancer patients $48.6( \pm 17.4)$ and for normal control it was 46 $( \pm 17.69)$, with a male to female ratio of 1:1 (Table 2).

\section{Region of gene deletion}

GSTM1 and GSTT1 gene deletions were mapped by sequencing the PCR products obtained with gene deletion primers. Sequencing results of GSTM1 gene revealed an amplified region $98 \mathrm{bp}$ upstream of the GSTM1 gene and a sequence of $293 \mathrm{bp}$ downstream of the gene. This indicated that the middle portion containing the entire GSTM1 gene was deleted, but promoter and other noncoding regions were present. The entire GSTM1 gene deletion spanning nearly $6 \mathrm{kbp}$ is shown in Figure 1. The amplified sequence for the GSTT1 gene deletion was mapped to $537 \mathrm{bp}$ up-

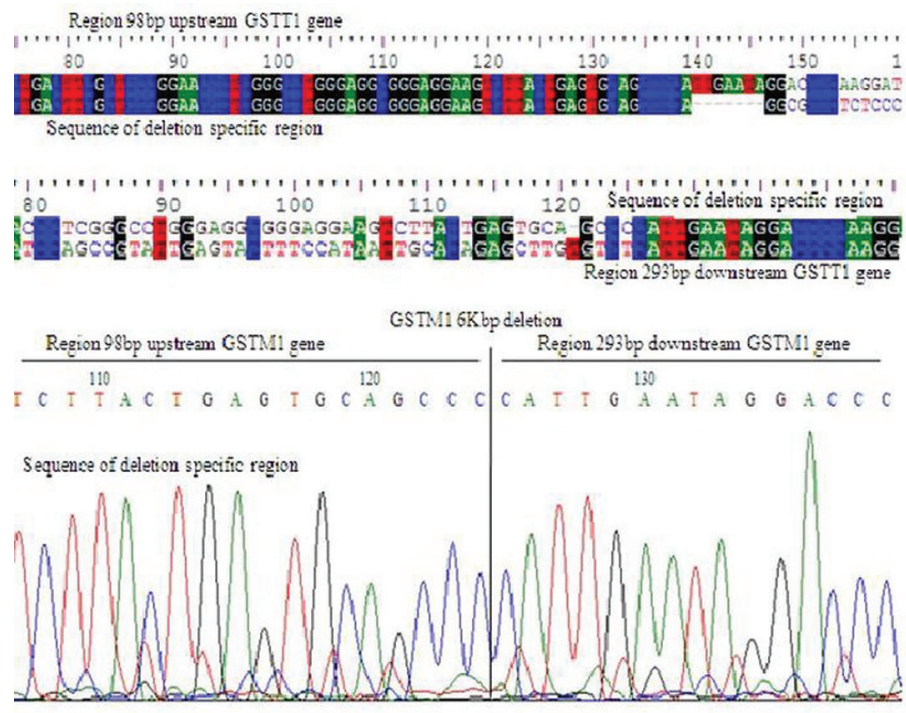

(a)

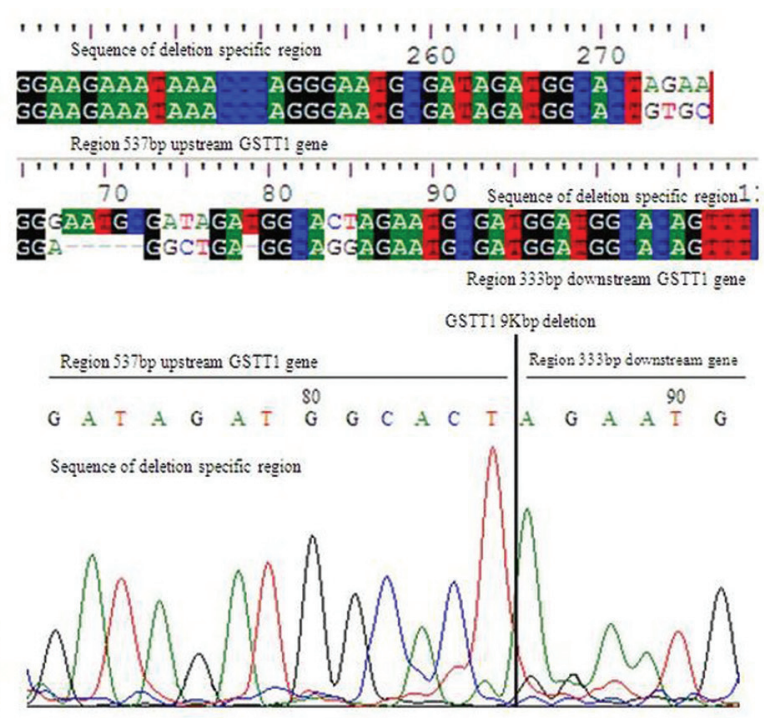

(b)

Figure 1 - Bioedit graph representing GSTM1 and GSTT1 gene deletions of approximately 6 kbp (a) and 9 kbp (b). The upstream and downstream intronic portions were present, but the complete gene-containing sequence was lacking. 
Table 2 - Table showing the demographic details of patients.

\begin{tabular}{lcc}
\hline & Cancer of pharynx & Cancer of larynx \\
\hline Male & 57 & 44 \\
Female & 43 & 48 \\
Age & $48.1( \pm 16.7)$ & $48.57( \pm 17.4)$ \\
Smoker & 54 & 47 \\
Non smoker & 48 & 45 \\
\hline
\end{tabular}

stream GSTT1 and 333 bp downstream of the GSTT1 gene. The total size of the GSTT1 deleted gene, along with some intronic portion was approximately $9 \mathrm{kbp}$ (Figure 1).

\section{The homozygous deletion genotypes}

A lack of amplification products for the GSTM1 or GSTT1 gene was considered as a homozygous deleted genotype (-/-). A total of 48 pharyngeal cancer patients out of 102, and 12 controls out of 150 had a GSTM1-deleted genotype (-/-). For laryngeal cancer patients, three out of 92 had a GSTM1 (-/-) genotype. Similarly, the GSTT1 deletion genotype (-/-) was found in 49 pharyngeal cancer patients out of 102, and in 15 laryngeal cancer patients out of 92, while 28 controls out of 150 also had a GSTT1 (-/-) genotype.

\section{The heterozygous genotypes}

The heterozygous genotype (+/-) for the GSTM1 gene was found in five out of 102 pharyngeal cancer patients and in four out of 92 laryngeal cancer patients. In the control group, 13 out of 150 had a heterozygous genotype $(+/-)$ for the GSTM1 gene. A heterozygous genotype (+/-) for the GSTT1 gene was detected in 12 pharyngeal cancer patients and in nine laryngeal cancer patients, compared to 19 controls.

\section{Association of homozygous deletions with cancers}

A significantly higher number of pharyngeal cancer patients had a GSTM1 gene deletion (-/-) compared with the controls. The odds ratio for the GSTM1 genotype (-/-) in patients compared to controls was 10.2 , with a $95 \% \mathrm{CI}$ of 5.04- 20.7. Similar results were found for laryngeal cancer patients, with significant associations (OR 0.5, CI 0.2-1.7). The OR for the homozygous GSTT1 gene deletion (-/-) in pharyngeal cancer cases compared to controls was 4.02 , with a $95 \% \mathrm{CI}$ of 2.3-7.1; for laryngeal cancer cases it was 0.8 , with a $95 \%$ CI of 0.4-1.7.

\section{Association of heterozygous genotypes with cancers}

For the GSTM1 gene an association with pharyngeal and laryngeal carcinoma was non-significant for the heterozygous genotype (+/-)in patients compared to controls (OR $0.5,95 \% \mathrm{CI} 0.2-1.6$ and $\mathrm{OR} 0.5,95 \% \mathrm{CI} 0.2-1.5$, respectively). Non- significance was also found for the GSTT1 gene when comparing pharyngeal (OR 0.9, 95\% CI
0.4-1.9) and laryngeal cancer (OR $0.7,95 \% \mathrm{CI} 0.3-1.7$ ) to controls (Table 3 ).

\section{Discussion}

In this case control study the genotyp characterization for the GSTM1 and GSTT1 genes was investigated. The study comprised three major aspects, viz mapping of GSTM1 and GSTT1 gene deletions, detecting of heterozygous and homozygous genotypes for the GSTM1 and GSTT1 genes, and investigating the association of these genotypes with the risk for developing laryngeal and pharyngeal cancer. Mapping showed deletions for the GSTM1 (6 kbp) and the GSTT1 genes (9 kbp). The heterozygous deletion genotype $(-/+)$ was higher in controls compared to patients. Homozygous deleted genotypes (-/-) were also observed for GSTM1GSTT1 the two genes. The frequency of $(-/-)$ genotypes was significantly higher $(\mathrm{p}<0.05)$ in pharyngeal and laryngeal cancer cases as compared to controls.

Earlier studies investigating the GSTM1 and GSTT1 genes did not map the exact location of the deletions (Toru et al., 2008). Even though PCR mapping and sequencing has been performed previously for GSTM1 and GSTT1 gene deletions (Kerb et al., 1999; Sprenger et al., 2000), a limitation of these studies was that they amplified a relatively large fragment of nearly 54,251 bp for the GSTT1 gene. To our knowledge, these assays have only been used in small scale studies because of the amount of work involved in genotyping such a large fragment (Buchard et al., 2007). Therefore, we devised a less cumbersome new method for PCR mapping by shortening fragment length. Multiple sets of primer were designed from noncoding sequences closer to the gene in order to minimize product fragment length.

The homozygous genotype status (-/-) has been investigated in different studies related to pharyngeal and laryngeal cancers (Oude et al., 2006; Cha et al., 2007). However, other studies also reports a lack of association with carcinomas (Hanna et al., 2001; Gajecka et al., 2005). But very few studies focussed on the heterozygous genotype of GSTM1 and GSTT1 gene deletions. Homozygous GSTM1 and GSTT1 deletions GSTM1GSTT1 result in the complete loss of respective enzymatic activity, whereas the presence of

Table 3 - Table showing the statistical details of polymorphisms found in pharyngeal and laryngeal cancer patients.

\begin{tabular}{|c|c|c|c|c|}
\hline Cancer/gene & & GSTM1 & & GSTT1 \\
\hline \multicolumn{5}{|c|}{ Homozygous deletion (-/-) } \\
\hline Pharynx & $47 \%$ & $10.2(5.04-20.7)$ & $48 \%$ & $4.02(2.3-7.1)$ \\
\hline Larynx & $3 \%$ & $0.5(0.2-1.7)$ & $16 \%$ & $0.8(0.4-1.7)$ \\
\hline \multicolumn{5}{|c|}{ Heterozygous deletion $(+/-)$} \\
\hline Pharynx & $5 \%$ & $0.5(0.2-1.6)$ & $12 \%$ & $0.9(0.4-1.9)$ \\
\hline Larynx & $4 \%$ & $0.5(0.2-1.5)$ & $10 \%$ & $0.7(0.3-1.7)$ \\
\hline
\end{tabular}


one functional allele in heterozygous genotypes is sufficient to confer some protective functions against carcinogens (Sprenger et al., 2000). A non-significant association of the heterozygous (GSTM1 and GSTT1) genotype in laryngeal and pharyngeal cancer patients is indicative of a compensatory role for the deleted allele. A significant number of pharyngeal and laryngeal cancer patients were found to be of the GSTM1 and GSTT1 homozygous deletion genotype when compared with controls. Similar results regarding GSTM1 gene deletions and laryngeal cancer, herein found in a Pakistani population, have also been reported in other populations, such as in Korea (Hong et al., 2000), Taiwan (Cheng et al., 2003), Turkey (Bardakci et al., 2003), and Poland (Gajecka et al., 2005). For GSTM1 gene deletions, a relation to pharyngeal cancer has been reported in Spain (Varela et al., 2008), Thailand (Tiwawech et al., 2005) and China (Xiuchan et al., 2008). Asian populations are reported to have the highest frequency for the GSTM1 and GSTT1 deletion genotype (Nosheen et al., 2010; Lee et al., 1995).

In conclusion, deletion of both GSTM1 and GSTT1 genes have been mapped and observed in relation to laryngeal and pharyngeal cancer. Carriers of GSTM1 and GSTT1 homozygous gene deletions are at an increased risk of developing these cancers in the Pakistani population as compared to heterozygous individuals. Further epidemiological studies and a larger sample size should provide a better understanding for the association of the homozygous and heterozygous deleted genotypes with pharyngeal and laryngeal cancer, for this carcinogen-metabolizing enzyme.

\section{Acknowledgments}

The authors would like to thank all the patients and individuals of the control group.. Research labs and equipments were provided by the COMSATS Institute of Information and Technology, Islamabad. The authors also acknowledge the financial support of Higher Education Commission, Islamabad (Pakistan).

\section{References}

Acar H, Kayhan O, Hamza MM, Selmon MY, Tulin C, Oguz C and Ozer B (2006) Relation of glutathione S transferase genotypes (GSTM1 and GSTT1) to laryngeal squamous cell carcinoma risk. Cancer Genet Cytol 169:89-93.

Bardakci F, Canbay E, Degerli N, Coban L and Canbay EI (2003) Relationship of tobacco smoking with GSTM1 gene polymorphism in laryngeal cancer. J Cell Mol Med 7:307-312.

Boccia S, Cadoni G, Sayed-Tabatabaei FA, Volante M, Arzani D, De Lauretis A, Cattel C, Almadori G, van Duijn CM, Paludetti G, et al. (2008) CYP1A1, CYP2E1, GSTM1, GSTT1, EPHX1 exons 3 and 4, and NAT2 polymorphisms, smoking, consumption of alcohol and fruit and vegetables and risk of head and neck cancer. J Cancer Res Clin Oncol 134:93-100.

Bongers V, Snow GB and Braakhuis BJ (1995) The role of glutathione $\mathrm{S}$-transferases in head and neck squamous cell carcinogenesis. Eur J Cancer B Oral Oncol 31B 6:349-354.
Buchard A, Juan JS, Kim D and Neils M (2007) Multiplex PCR detection of GSTM1, GSTT1 and GSTP1 gene variants. J Mol Diagnost 9:612-617.

Cha IH, Park JY, Chung WY, Choi MA, Kim HJ and Park KK (2007) Polymorphisms of CYP1A1 and GSTM1 genes and susceptibility to oral cancer. Yonsei Med J 48:233-239.

Cheng L, Sturgis E and Eicher S (1999) Glutathione S-transferase polymorphisms and risk of squamous cell carcinoma of the head and neck. Int J Cancer 84:220-224.

Cheng YJ, Chien YC, Hildesheim A, Hsu MM, Chen IH, Chuang J, Chang J, Ma YD, Luo CT, Hsu WL, et al. (2003) No association between genetic polymorphisms of CYP1A1 GSTM1, GSTT1, GSTP1, NAT2, and nasopharyngeal carcinoma in Taiwan. Cancer Epidemiol Biomarkers Prev 12:179-180.

Deng ZL, Wei YP and Ma Y (2004) Frequent genetic deletion of detoxifying enzyme GSTM1 and GSTT1 genes in nasopharyngeal carcinoma patients in Guangxi Province, China. Zhonghua Zhong Liu Za Zhi 26:598-600.

Gajecka M, Rydzanicz M, Jaskula-Sztul R, Kujawski M, Szyfter W and Szyfter K (2005) CYP1A1, CYP2D6, CYP2E1, NAT2, GSTM1, and GSTT1 polymorphisms or their combinations are associated with the increased risk of the laryngeal squamous cell carcinoma. Mutat Res 574:112-123.

Hanna E, MacLeod S, Vural E and Lang N (2001) Genetic deletions of glutathione $\mathrm{S}$ glutathione as a risk factor in squamous cell carcinoma of the larynx: A preliminary report. Am J Otolaryngol 22:121-123.

Hong YJ, Lee JK, Lee GH and Hong SI (2000) Influence of glutathione $\mathrm{S}$ transferase $\mathrm{M} 1$ and $\mathrm{T} 1$ genotypes on larynx cancer risk among Korean smokers. Clin Chem Lab Med 38:917-919.

Kerb R, Brockmoller J, Sachse C and Roots I (1999) Detection of the GSTM $1 * 0$ allele by long polymerase chain reaction. Pharmacogenetics 9:89-94.

Khan MI, Micheal S, Akhtar F, Ahmed W, Ijaz B, Ahmed and Qamar R (2010) The association of glutathione S transferase GSTT1 and GSTM1 gene polymorphism with pseudoexfoliative glaucoma in a Pakistani population. Mol Vis 16:2146-2152.

Lee EJ, Wong JY, Yeoh PN and Gong NH (1995) Glutathione S transferase theta (GSTT1) genetic polymorphism among Chinese, Malays and Indians in Singapore. Pharmacology 5:332-334.

Lin CL, Lo WF, Lee TH, Ren Y, Hwang SL, Cheng YF, Chen CL, Chang YS, Lee SP, Rickinson AB, et al. (2002) Immunization with Eppstein Barr Virus (EBV) peptide-pulsed dendritic cells induces functional CD8 $+\mathrm{T}$ cell immunity and may lead to tumor regression in patients with EBV- positive nasopharyngeal carcinoma. Cancer Res 62:6952-6958.

Nosheen M, Ishrat M, Malik FA, Baig RM and Kayanai MA (2010) Association of GSTM1 and GSTT1 gene deletions with risk of head and neck cancer in Pakistan: A case control study. Asian Pacif J Cancer Prev 11:1-6.

Oude OM, Manni JJ and Peters WHM (2006) Glutathione S transferase T1 null polymorphism and the risk for head and neck cancer. Acta Otolaryngol 126:311-317.

Pemble S, Schroeder K and Spencer S (1994) Human glutathione S-transferase theta (.GSTT1): cDNA cloning and the characterization of a genetic polymorphism. Biochem J 300:271-276. 
Shaikh RS, Amir M, Masood AI, Sohail A, Athar HU, Siraj S, Ali M and Anjam MS (2010) Frequency distribution of GSTM1 and GSTT1 null allele in Pakistani population and risk of disease incidence. Environ Toxicol Pharmacol 30:76-79.

Sinnett D, Krajinovic M and Labuda D (2000) Genetic susceptibility to childhood acute lymphoblastic leukemia. Leuk Lymphoma 38:447-462.

Sprenger R, Schlagenhaufer R, Kerb R, Bruhn C, Brockmöller J, Roots I and Brinkmann U (2000) Characterization of the glutathione S-transferase GSTT1 deletion: Discrimination of all genotypes by polymerase chain reaction indicates a trimodular genotype-phenotype correlation. Pharmacogenetics 10:557-565.

Tiwawech D, Srivatanakul P, Karalak A and Ishida T (2005) Glutathione S transferase M1 gene polymorphism in Thai nasopharyngeal carcinoma. Asian Pac J Cancer Prev 6:270-275.

Toru H, Masaharu Y, Shinji T and Kazuaki C (2008) Genetic polymorphisms and head and neck cancer risk. Int J Oncol 32:945-973.

Varela L, Ravina AR, Crespo MAJ, Kelsey KT, Loidi L and Dios JMB (2008) CYP1A1, mEH, and GSTM1 polymorphisms and risk of oral and pharyngeal cancer: A Spanish casecontrol study. J Oncol doi:10.1155/2008/741310.

Xiuchan G, Stephen JOB, Yi Z, Nelson GW and Winkler CA (2008) GSTM1 and GSTT1 gene deletions and the risk for nasopharyngeal carcinoma in Han Chinese. Cancer Epidemiol Biomarkers Prev 17:1780.

Associate Editor: Anamaria Aranha Camargo

License information: This is an open-access article distributed under the terms of the Creative Commons Attribution License, which permits unrestricted use, distribution, and reproduction in any medium, provided the original work is properly cited. 